\title{
Sectoral H-plane SBFSS-SIW Horn Antenna
}

\author{
N. Esparza, P. Alcón, L. F. Herrán and F. Las-Heras \\ Signal and Communications Theory Area, Department of Electrical Engineering, University of Oviedo \\ E-33203, Gijón, Spain \\ \{nesparza, palcon, lfherran, flasheras\} @ tsc.uniovi.es
}

\begin{abstract}
A sectoral H-plane SIW horn antenna based on Stop-Band Frequency Selective Surface Substrate Integrated Waveguide technology is presented. The FSS operating in StopBand that replace the original metal conductor plates of the horn, increase the versatility of these structures, by making the direct integration of biased active and passive devices on its surface possible. The return losses, the radiation patterns and the E-field distribution on the surface of the proposed antenna have been compared with those of its counterpart SIW horn, to demonstrated that the new antenna can be used in the same role as the SIW horn with the advantage of having electrically isolated pads from ground on its surface.
\end{abstract}

\section{INTRODUCTION}

Since the rectangular waveguide horns development in the late $1800 \mathrm{~s}$, it is certainly one of the most widely employed microwave antennas. Although its use was focused on microwaves and waveguide transmission lines in its early period, nowadays its applicability has been widespread, as consequence of its high potential: simplicity in construction, ease of excitation, symmetric patterns, relatively high gain, directivity performance, wide bandwidth and versatility [1]. The development of substrate integrated waveguide technique in the 2000s solved their incompatibility with the essential properties required for the successful design of microwave and millimetre-wave systems: low cost, mass production, highperformance and high-yield [2]. Nevertheless, the electrical connection of the SIW metal layers to ground challenges the direct integration of biased active/passive devices in SIW structures. Recent research have proposed the combination of SIW technology with FSS structures operating in their stopband to solve this drawback, preserving the original SIW propagation properties and dimensions [3].

In this paper, the implementation of a SIW horn antenna based on SBFSS-SIW technology that incorporates periodic isolated pads from ground on its top and bottom metal layers is presented. Firstly, the topology of the antenna and its procedure design are presented. Secondly, the experimental results obtained are compared with those of its counterpart SIW horn, and illustrated to demonstrate that the proposed SBSFSS-SIW horn can be used in the same role as a SIW horn.

\section{Antenna DESIGN}

The designed antenna is a three-layer sectoral H-plane SBFSS-SIW horn with phase correction that operates in the Xband. A sketch of its internal configuration and dimensions are schematically shown in Fig. 1. It is based on SIW technology,

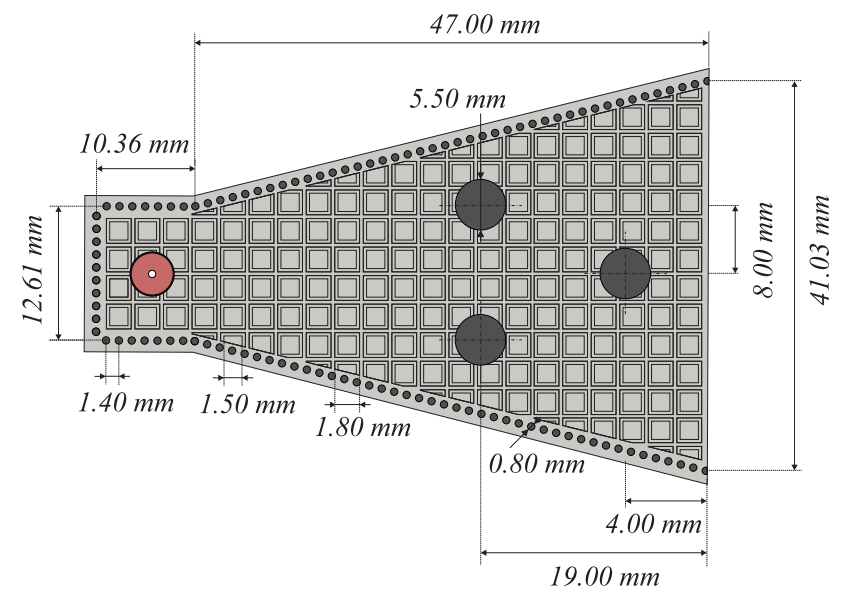

Fig. 1: Schematic of the proposed SBFSS-SIW horn antenna.

so its side walls are composed by a set of metallized cylindrical via-holes [4]. The feed rectangular waveguide behaves as a filled-dielectric WR-90 standard waveguide, prepared to propagate only the dominant $T E_{10}$ mode in the operation frequency range of the structure. A coaxial feeding has been used in order to eliminate spurious radiation due to feed network. The flared section, designed following the same principles as normal horn antennas [1], includes inductive posts as phase correctors [5]. Their dimensions and allocation have been adjusted according to the requirements of the problem to solve. The top and bottom metal plates of the structure have been replaced by square periodic FSS cells with a stop-band response in the operation frequency range of the antenna and the resonance frequency allocated at least twice over the frequency operation range of the antenna to not disturb its behavior as a SIW horn [3]. The equations presented in [6] were used to calculate the preliminary dimensions of the square cells, and their final value was optimized to meet the desired frequency response, taking into account the limitations of the laser micromachining employed and the minimum pad size to connect external devices on the FSS cells of the metal plates of the antenna. The antenna was manufactured using a standard 60 mil RO4003C substrate.

\section{EXPERIMENTAL RESULTS}

The return losses measured in the antenna including the parasitics effects of the coaxial connector used are illustrated in Fig. 2. In adittion to the operation bandwidth $\left(\left|S_{11}\right|<-10\right.$ 
$\mathrm{dB}$ ) of both antennas, which is on the order of $200 \mathrm{MHz}$, a widened frequency range is plotted to show the lightly effects the geometry and location of the FSS on the scattering parameters of the structure [3].

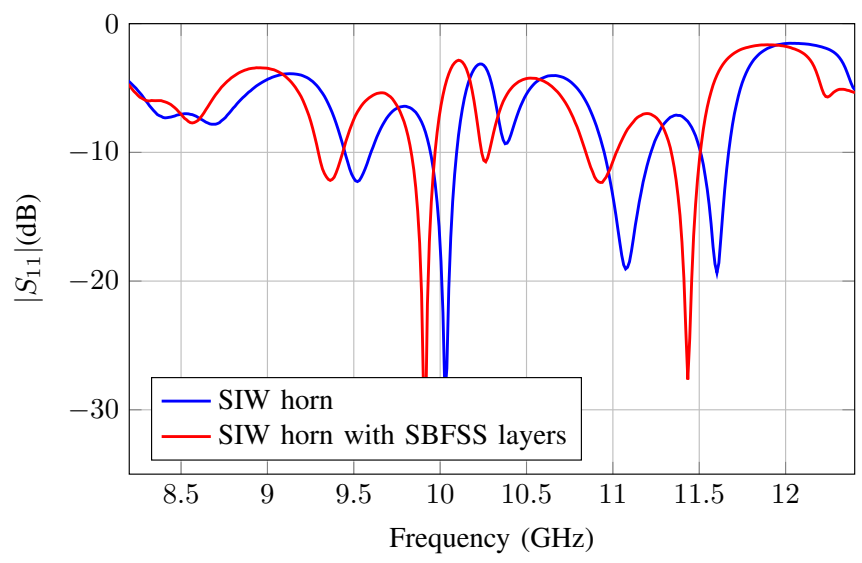

Fig. 2: Measured return losses of the proposed SBFSS-SIW horn and its counterpart in SIW (In color).

The equivalent $E$-field distribution associated to the $\mathrm{TE}_{10}$ inside the SBFSS-SIW horn and SIW horn are plotted in Figs. $3 \mathrm{a}$ and $3 \mathrm{~b}$ respectively. The powerful red colour represents the maximum level of $E$-field propagated inside the H-plane horns at $10.3 \mathrm{GHz}$ and the strong blue the minimum level. These results demonstrate that both structures behave in a similar way, so the topology of the SBFSS-SIW horn doesn't internally modify the original structure. The $E$-plane and $H$ plane radiation patterns for different frequencies are shown in Figs. 4 and 5. They are normalized to the maximum value of the SIW horn antenna in broadside direction. The resemblance between these results demonstrate the viability of the integration of FSS on the metal surfaces without disturbing the initial propagation characteristics of the horn.

\section{CONCLUSION}

A sectoral H-plane SIW horn antenna implemented using the SBFSS-SIW technology is presented. Its top and bottom metal plates have been replaced by periodic pads electrically isolated from ground. The results illustrated in this paper demonstrate the feasibility of design SIW horns maintaining their original dimensions and propagation properties but with the improvement of having pads on its surface that allow the direct integration of devices on it, a property that increases its applicability.

\section{ACKNOWLEDGEMENT}

This work was supported by the Gobierno del Principado de Asturias under the Plan de Ciencia Tecnología e Innovación (PCTI) by Grants BP13042 and BP12032, by the Gobierno del Principado de Asturias and FEDER (Unión Europea) under Project GRUPIN14-114, and by the Ministerio de Economía y Competitividad under Project TEC2014-54005-P.

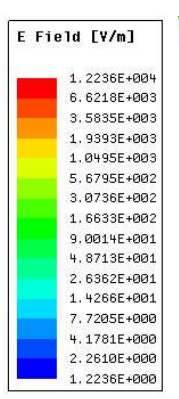

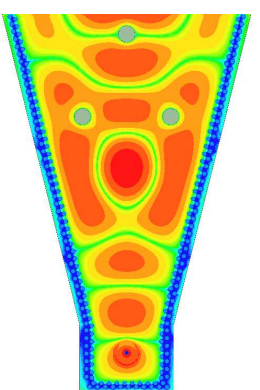

(a)

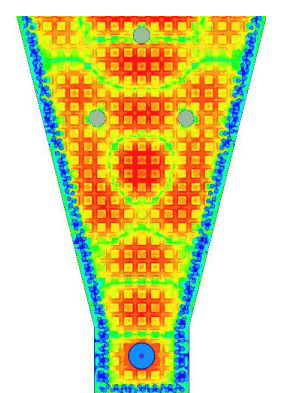

(b)
Fig. 3: $E$-field distribution of dominant mode at $10.3 \mathrm{GHz}$ inside the: (a) SBFSS-SIW horn and (b) SIW horn.

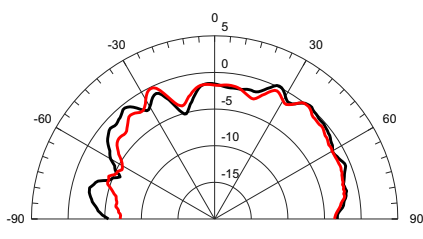

(a)

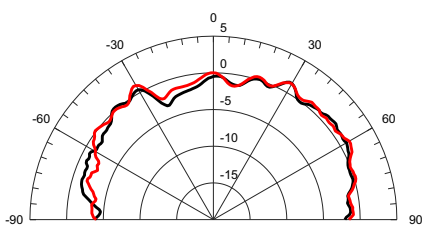

(b)
Fig. 4: Measured normalized radiation pattern (E-plane) of SBFSS-SIW horn (black solid line) and SIW horn (red solid line) at: (a) $10.7 \mathrm{GHz}$ and (b) $11.25 \mathrm{GHz}$.

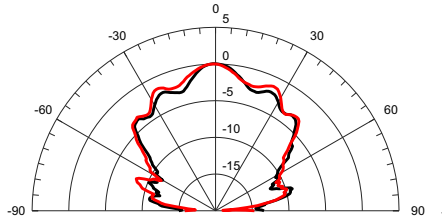

(a)

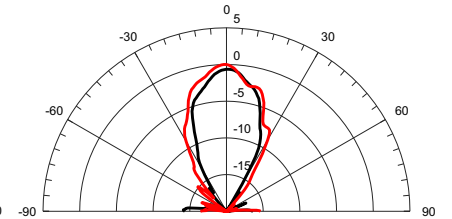

(b)
Fig. 5: Measured normalized radiation pattern (H-plane) of SBFSS-SIW horn (black solid line) and SIW horn (red solid line) at: (a) $10.7 \mathrm{GHz}$ and (b) $11.25 \mathrm{GHz}$.

\section{REFERENCES}

[1] Balanis, C. A., Antenna Theory - Analysis and Design, John Wiley \& Sons, Inc., Hoboken, New Jersey, 2005.

[2] Wu, K., "Towards system-on-substrate approach for future millimeterwave and photonic wireless applications," Asia-Pacific Microwave Conference, 1895-1900, 2006.

[3] N. Esparza et al., "Substrate Integrated Waveguides Structures Using Frequency Selective Surfaces Operating in Stop-Band (SBFSS-SIW)," IEEE Microwave and Wireless Components Letters, 2016.

[4] Bozzi, M. et al. , "Review of substrate-integrated waveguide circuits and antennas," Microwaves, Antennas \& Propagation, IET, Vol. 5, 909-920, 2011.

[5] Wang, L. et al., "Phase corrected substrate integrated waveguide h-plane horn antenna with embedded metal-via arrays," IEEE Transactions on Antennas and Propagation, Vol. 62, 1854-1861, 2014.

[6] B. A. Munk, Frequency Selective Surfaces, N. Y. Wiley, Ed., 2000. 